\title{
What is the Function of Hippocampal Theta Rhythm?- Linking Behavioral Data to Phasic Properties of Field Potential and Unit Recording Data
}

\author{
Michael E. Hasselmo*
}

\begin{abstract}
The extensive physiological data on hippocampal theta rhythm provide an opportunity to evaluate hypotheses about the role of theta rhythm for hippocampal network function. Computational models based on these hypotheses help to link behavioral data with physiological measurements of different variables during theta rhythm. This paper reviews work on network models in which theta rhythm contributes to the following functions: (1) separating the dynamics of encoding and retrieval, (2) enhancing the context-dependent retrieval of sequences, (3) buffering of novel information in entorhinal cortex (EC) for episodic encoding, and (4) timing interactions between prefrontal cortex and hippocampus for memory-guided action selection. Modeling shows how these functional mechanisms are related to physiological data from the hippocampal formation, including (1) the phase relationships of synaptic currents during theta rhythm measured by current source density analysis of electroencephalographic data from region CA1 and dentate gyrus, (2) the timing of action potentials, including the theta phase precession of single place cells during running on a linear track, the contextdependent changes in theta phase precession across trials on each day, and the context-dependent firing properties of hippocampal neurons in spatial alternation (e.g., "splitter cells"), (3) the cholinergic regulation of sustained activity in entorhinal cortical neurons, and (4) the phasic timing of prefrontal cortical neurons relative to hippocampal theta rhythm. 2005 Wiley-Liss, Inc.
\end{abstract}

KEY WORDS: EEG oscillations; region CA3; region CA1; spatial alternation; fornix; septum; theta phase precession; computational modeling

\section{INTRODUCTION}

Electroencephalographic recordings from the hippocampus and related structures show a prominent, large amplitude oscillation termed theta rhythm, which normally appears with a frequency of 6-7 oscillations/s (Green and Arduini, 1954; Vanderwolf, 1969; Vanderwolf et al., 1977; Buzsáki et al., 1983; Stewart and Fox, 1990; Bland and Colom, 1993; Buzsáki, 2002). This falls within the 4-7 Hz range labeled as "theta" in human EEG, but the definition has been expanded in animals to include higher frequencies that appear during running

Department of Psychology, Center for Memory and Brain, Program in Neuroscience, Boston University, Boston, Massachusetts

Grant sponsor: NIMH; Grant numbers: 60013, 61492, 60450; Grant sponsor: NSF-Science of Learning Center; Grant number: SBE 0354378; Grant sponsor: Collaborative Research in Computational Neuroscience (CRCNS); Grant number: NIDA 16454.

*Correspondence to: Michael E. Hasselmo, Department of Psychology, Center for Memory and Brain, Program in Neuroscience, Boston University, 2 Cummington St., Boston, MA 02215. E-mail: hasselmo@bu.edu

Accepted for publication 17 June 2005

DOI 10.1002/hipo.20116

Published online 12 September 2005 in Wiley InterScience (www.interscience. wiley.com).
(Fox et al., 1986; Bragin et al., 1995) as well as the low frequencies of $3-4 \mathrm{~Hz}$ that appear in urethaneanesthetized rats and during Type II atropine-sensitive theta (Kramis et al., 1975; Fox et al., 1986; Bland and Colom, 1993; Wyble et al., 2000). Considerable research has focused on the mechanisms of theta rhythm, but the focus of this paper is the possible function of theta rhythm: the link between physiological data and behavioral function.

This paper reviews a series of computational models (Hasselmo et al., 2002b; Hasselmo and Eichenbaum, 2005) that address the question: How are specific behavioral functions of hippocampal circuits enhanced by oscillations of physiological variables in the theta frequency range? Recent models in this series directly simulate performance of a virtual rat in behavioral tasks such as spatial alternation. Rats show impairments in such tasks with lesions or inactivation of the fornix and medial septum (Givens and Olton, 1990; Aggleton et al., 1995; Ennaceur et al., 1996), which also cause strong reductions in hippocampal theta rhythm (Rawlins et al., 1979; Buzsáki et al., 1983). The models guide behavior in these tasks using biologically based network simulations, which address features of physiological data on the theta rhythm. This review will first address behavioral data, then summarize relevant physiological data, and conclude with models showing the link between physiology and behavior.

\section{BEHAVIORAL DATA}

Historically, behavioral data have been used to support a role for theta rhythm in one of two main behavioral functions: (1) voluntary movement, or (2) learning and memory. Researchers such as Vanderwolf performed studies correlating theta rhythm with specific behavioral states, and argued that theta rhythm was particularly prominent in association with voluntary movement (Vanderwolf, 1969; Whishaw and Vanderwolf, 1973; Bland and Oddie, 2001). This movement includes free running (O'Keefe and Nadel, 1978; Skaggs et al., 1996) as well as running in a running wheel (Buzsáki et al., 1983; Hyman et al., 2003) or on a treadmill (Fox et al., 1986; Brankack et al., 1993). The phase of theta rhythm also appears to correlate with the phase of motor output, including the 
timing of sniffing (Macrides et al., 1982), and the timing of whisker movement (Semba and Komisaruk, 1984; Lerma and Garcia-Austt, 1985). Considerable data support a role of theta rhythm in the sensory-motor interface (Bland and Oddie, 2001). However, theta rhythm (albeit at lower frequencies) also appears during immobility in rats and mice during fear conditioning (Whishaw, 1972; Sainsbury et al., 1987a; Seidenbecher et al., 2003), or attention to predators (Sainsbury et al., $1987 \mathrm{~b})$. Theta rhythm also shows up prominently in immobile rabbits (Berry and Seager, 2001; Seager et al., 2002; Griffin et al., 2004) during both aversive eyeblink conditioning and appetitive conditioning.

Considerable research has also focused on the correlation of theta rhythm with learning and memory (Berry and Thompson, 1978; Winson, 1978; Givens and Olton, 1990; Vertes and Kocsis, 1997; Berry and Seager, 2001). An early study showed that the impairment in a spatial memory task caused by lesions of the medial septum was correlated with the amount of reduction of the hippocampal theta rhythm (Winson, 1978). Lesions of the medial septum and fornix reduce hippocampal theta power (Rawlins et al., 1979) and cause impairments in a number of memory-guided tasks, including spatial alternation (Givens and Olton, 1990; Aggleton et al., 1995; Ennaceur et al., 1996), delayed nonmatch to position (Markowska et al., 1989), operant delayed alternation (Numan and Quaranta, 1990), and spatial reversal (M'Harzi et al., 1987). The impairments appear specific to recent, episodic memory, as fornix lesions do not impair the initial learning of a goal location, but impair the learning of reversal (M'Harzi et al., 1987), and medial septal inactivation does not impair reference memory, but impairs recent episodic memory in continuous conditional discrimination (Givens and Olton, 1994). The role in learning is supported by extensive data in rabbits. The rate of learning is faster in individual rabbits when the hippocampal EEG has the highest amount of theta power (Berry and Thompson, 1978). When delivery of the conditioned stimulus is timed to appear during periods of theta rhythm, the rate of conditioning to the stimulus is enhanced in both delay conditioning (Seager et al., 2002) and trace conditioning (Griffin et al., 2004). Theta rhythm appears to reset its phase for encoding new stimuli during presentation of visual stimuli in a delayed match to sample task (Givens, 1996) but not during a reference memory task, and this phase resetting allows enhanced induction of longterm potentiation (LTP) (McCartney et al., 2004). Phase resetting shows specificity for item encoding vs. retrieval probe phases in human memory tasks (Rizzuto et al., 2003), suggesting a role for phase reset in determining appropriate dynamics for encoding and retrieval.

Although the categories of voluntary movement and learning and memory appear to be mutually exclusive, the models reviewed here can link behavior to physiology without assigning such categories. In these models, performance of memoryguided behavior requires an ongoing interaction of motor selection processes and memory retrieval that require theta rhythm for the synchronization and integration of both of these apparently separate processes.

\section{PHYSIOLOGICAL DATA}

The behavioral data suggest a role for theta rhythm in behavior, but it is not yet clear how these specific behavioral functions are enhanced by oscillations of physiological properties of neurons and networks in the theta frequency range. Modeling demonstrates how behavioral function in these tasks could depend upon specific physiological phenomena associated with theta rhythm. These physiological data on theta rhythm oscillations within the hippocampal formation are reviewed in this section, and are grouped according to the four hypotheses analyzed in the model: (1) separation of encoding and retrieval, (2) context-dependent retrieval of sequences, (3) buffering in entorhinal cortex (EC), and (4) timing of interactions with prefrontal cortex.

In describing the link to data, it is important to note the different conventions for describing the phase of theta rhythm. Many studies focus on EEG recording at the location of largest amplitude theta rhythm, at the border between stratum lacunosum-moleculare of hippocampal region CA1, and stratum moleculare of the dentate gyrus - a location known as the hippocampal fissure. Some studies use the peak of the fissure EEG as phase zero (Fox et al., 1986). However, unit recording studies often use theta rhythm recorded in stratum pyramidale, which has a phase $\sim 180^{\circ}$ different from fissure EEG (Buzsáki et al., 1983, 2002; Leung, 1984; Skaggs et al., 1996; Csicsvari et al., 1999). Here, we will sometimes use the convention used in unit recording studies, with reference phase zero near the peak of theta in stratum pyramidale (near the trough of theta in the fissure EEG). It is worth noting that the unfiltered EEG during theta rhythm does not have a sinusoid shape, but has a scalloped shape (Buzsáki, 2002) that could be more effective at providing linear read-out of a sequence (Hasselmo and Eichenbaum, 2005).

\section{Data Relevant to Separation of Encoding and Retrieval}

A range of experiments have demonstrated phasic changes in physiological variables during theta rhythm. These data support models that focus on separate dynamics of encoding and retrieval during different phases of each theta rhythm cycle (Hasselmo et al., 2002b).

\section{Current source density studies of synaptic transmission}

Theta rhythm is associated with laminar segregation of rhythmic sources and sinks in region CA1 and dentate gyrus, as described using current source density analysis of EEG data (Buzsáki et al., 1986; Brankack et al., 1993), and summarized in Figure 1. This current source density analysis supports the model of separate phases of encoding and retrieval. The strongest input from EC occurs at the trough of fissure EEG, when there are prominent sinks in stratum lacunosum-moleculare (Brankack et al., 1993). This is proposed as the encoding phase 

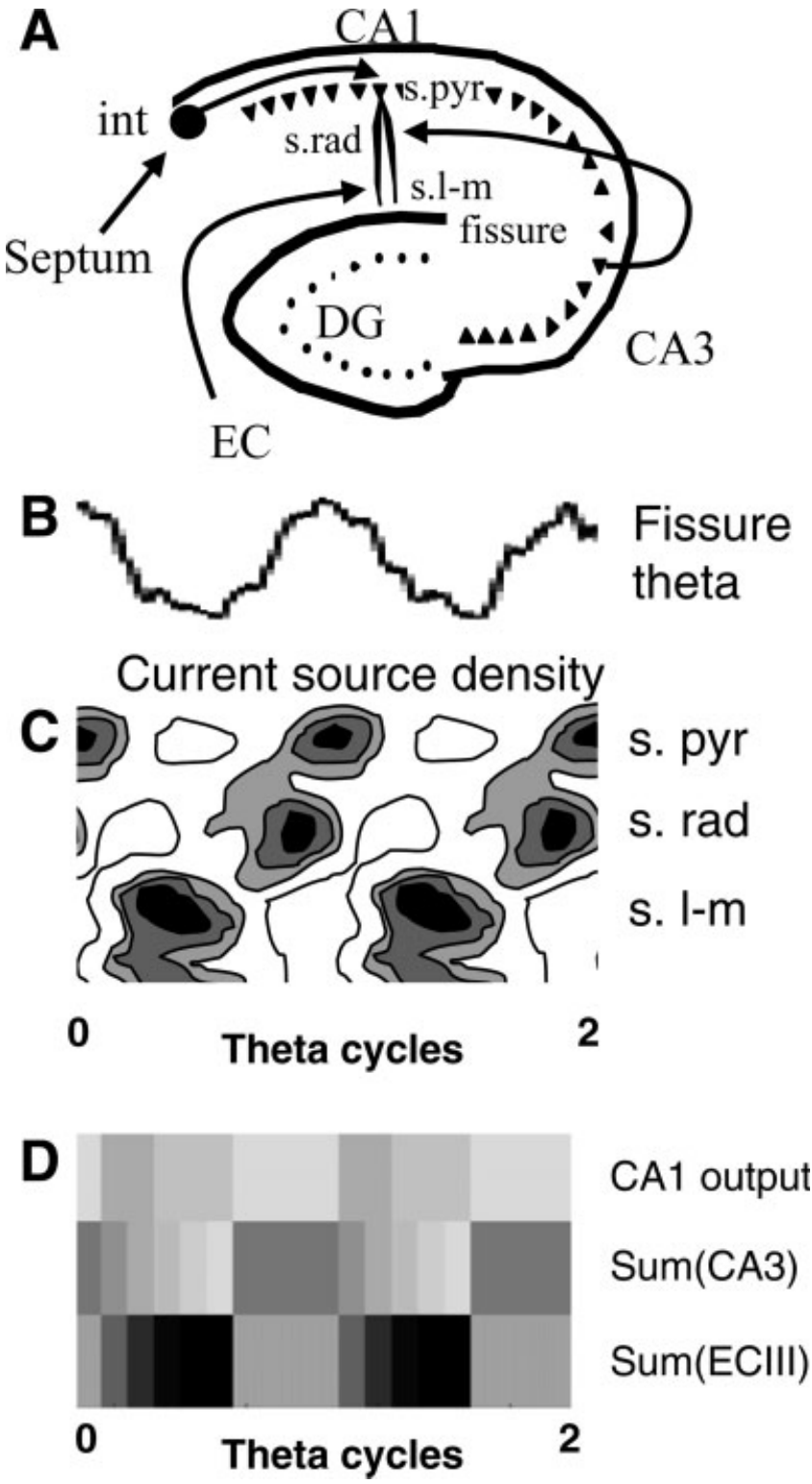

FIGURE 1. A: Anatomy of hippocampus showing synaptic inputs to region CA1 that contribute to theta rhythm oscillations in the EEG. These include input from EC to stratum lacunosum-moleculare (s.l-m), input from region CA3 to stratum radiatum (s. rad) and input from medial septum to inhibitory interneurons (int) in stratum pyramidale (s. pyr). B: Example of theta rhythm oscillation in the EEG recorded at the hippocampal fissure. C: Current source density data adapted from Brankack, Stewart, and Fox (1993), showing rhythmic changes in current sinks because of synaptic input to different layers of region CA1. Darker colors indicate stronger inward current sinks. Current sinks at top indicate summed currents in region CA1 s.pyr, middle sinks are caused by synaptic input from region CA3 to s. rad, and bottom sinks are caused by synaptic input from ECIII to $s .1-\mathrm{m}$. D: Simulation of theta rhythmic activity (Hasselmo and Eichenbaum, 2005). Each band shows the sum of rhythmic activity in modeled subregions across two cycles of simulation time plotted horizontally. Darker colors indicate stronger activity. The top band shows the sum of region CA1 output, the middle band shows the sum of region CA3 activity, and the bottom band shows sum of EC layer III activity.
(Fig. 2). This strong input to the dendrites could allow encoding at a time when there is little retrieval spiking activity in the pyramidal cell layer, which at this phase shows prominent outward currents in stratum pyramidale (Brankack et al., 1993) because of strong inhibition at the cell body (Kamondi et al., 1998). In contrast, the peak of fissure EEG (the retrieval phase) is associated with prominent sinks in stratum radiatum and another sink in stratum pyramidale, associated with the greater firing of CA1 pyramidal cells at the peak of the fissure EEG (Fox et al., 1986; Skaggs et al., 1996; Csicsvari et al., 1999). As shown in Figure 2, the peak of fissure EEG is assumed to correspond to the retrieval phase of network function (Hasselmo et al., 2002b).

\section{Phasic changes in synaptic transmission}

The phasic changes in current sinks could arise from differences in presynaptic firing rate, but could also arise from differences in presynaptic inhibition of synaptic transmission (Wyble et al., 2000). Recording of evoked synaptic potentials at different phases of theta rhythm demonstrates a consistent change in magnitude of the rising slope of synaptic potentials (Wyble et al., 2000) as well as in the magnitude of evoked population spikes (Rudell et al., 1980, 1984; Buzsaki et al., 1981). The change in magnitude of synaptic transmission could be due to phasic changes in presynaptic inhibition caused by $\mathrm{GABA}_{B}$
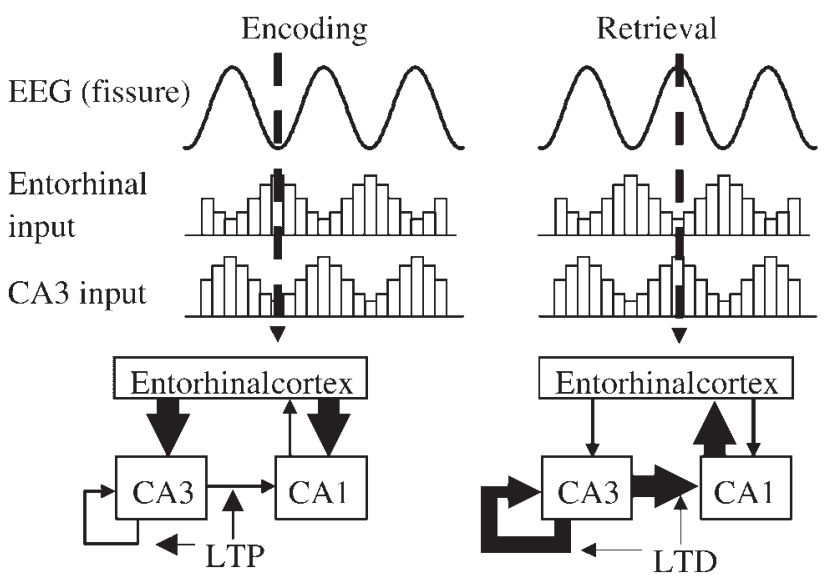

Strong entorhinal input Weak CA3 input Strong LTP

Weak entorhinal input Strong CA3 input LTD or depotentiation

FIGURE 2. Separation of encoding and retrieval during theta rhythm (Hasselmo et al., 2002b). LEFT: The encoding phase is at the trough of fissure theta, when synaptic currents arising from EC are strong (Brankack et al., 1993), because of greater depolarization of ECIII and greater spread of activity in that structure. Transmission from CA3 is weak (Wyble et al., 2000), preventing retrieval, but LTP in these synapses is very strong (Holscher et al., 1997; Hyman et al., 2003), allowing encoding of associations between EC inputs. RIGHT: The retrieval phase is at the peak of fissure theta, when synaptic currents arising from EC are weak, but synaptic currents arising from CA3 are strong (Brankack et al., 1993), allowing effective retrieval of previously encoded sequences. During this phase synapses do not encode the retrieval because they do not show LTP, instead they show LTD or depotentiation. 
receptors (Hasselmo and Fehlau, 2001), which appears to have a sufficiently rapid time course in vivo to cause this effect (Molyneaux and Hasselmo, 2002). As discussed later, these phasic changes could cause synaptic transmission in stratum radiatum to be weak when induction of LTP is strong during encoding, and then allow strong transmission during retrieval, when LTP is weak (Hasselmo et al., 2002b).

\section{Phasic changes in membrane potential}

Another factor contributing to the change in magnitude of transmission and population spikes is the postsynaptic membrane potential of pyramidal cells in regions CA1 and CA3. Intracellular recordings have demonstrated phasic changes in pyramidal cell depolarization during theta rhythm (Fujita and Sato, 1964; Fox, 1989; Kamondi et al., 1998). In particular, the soma membrane potential appears to be hyperpolarized at the time when dendrites are receiving the strongest depolarizing current input from EC (Kamondi et al., 1998). As discussed later, this hyperpolarization could prevent interference because of retrieval of previously stored associations during encoding of new associations (Hasselmo et al., 2002b).

\section{Induction of LTP}

The separation of encoding and retrieval in these models depends upon phasic changes in the induction of LTP during theta rhythm. Initially, it was shown in urethane-anesthetized rats that LTP is more effectively induced in the dentate gyrus when a tetanus is delivered on positive phases of theta (Pavlides et al., 1988), and similar results have been shown in freely moving animals (Orr et al., 2001). Similar effects appear in region CA1. In slice preparations showing theta rhythm due to cholinergic agonists, stimulation on the peak of theta recorded locally in stratum radiatum causes LTP, while stimulation on the trough causes long-term depression (LTD) (Huerta and Lisman, 1995). In urethane-anesthetized animals, stimulation delivered at the peak of the theta wave recorded locally in stratum radiatum induces LTP (Holscher et al., 1997), while stimulation delivered at negative phases of theta causes depotentiation. Note that the local peak in stratum radiatum is phase shifted from fissure EEG, and would be closer to the encoding phase at the trough of fissure EEG. Recently, induction of LTP in region CA1 was analyzed in awake, behaving animals (Hyman et al., 2003), showing that stimulation on the peak of local theta induces LTP, while stimulation on the trough induces LTD (see Fig. 2). These results suggest that induction of LTP in stratum radiatum occurs when transmission is weak but dendrites are depolarized by entorhinal input. LTP does not depend on spiking at the soma, as dendritic spikes can induce LTP even when the soma is hyperpolarized (Golding et al., 2002).

\section{Firing of hippocampal units relative to theta rhythm}

Modeling has addressed some of the data concerning the preferred phase of action potential firing by different neuronal populations (Fox et al., 1986; Brankack et al., 1993), which provides an extensive database for fitting of functional models. Hippocampal pyramidal cells tend to fire most frequently near the positive peak of the fissure EEG, while showing less firing activity in phases near the trough (Fox et al., 1986; Skaggs et al., 1996; Csicsvari et al., 1999). Inhibitory interneurons fire at a different phase. In anesthetized animals, the firing of interneurons appears to be maximal at the negative phase of fissure theta $-180^{\circ}$ from the peak (Buzsáki and Eidelberg, 1983; Fox et al., 1986), whereas in awake, behaving animals, firing of interneurons appears to precede the peak of fissure theta by about $50^{\circ}$ (Fox et al., 1986; Skaggs et al., 1996; Csicsvari et al., 1999). This phase of interneuron firing has been simulated in detailed biophysical simulations of the hippocampal formation (Kunec et al., 2005). In this simulation, the phase of firing of one class of interneurons, the orienslacunosum-moleculare (O-L-M) cells, might allow selective inhibition of entorhinal input to lacunosum-moleculare at a time when pyramidal cell firing should be dominated by stratum radiatum input.

\section{Data Relevant to Context-Dependent Retrieval}

The aforementioned data have been used in support of the separation of encoding and retrieval. Recent models have focused on the dynamics of retrieval, and mechanisms allowing selective context-dependent retrieval of sequences (Hasselmo and Eichenbaum, 2005). This model addresses the following data.

\section{Theta phase precession}

Hippocampal place cells show the phenomenon of theta phase precession, firing late in the theta cycle when a rat first enters the place field of the cell, and firing at earlier phases as the rat moves through the place field (O'Keefe and Recce, 1993; Skaggs et al., 1996; Mehta et al., 2002; Huxter et al., 2003), as shown in Figures 3 and 4. Retrieval of sequences has been used to model the phenomenon of theta phase precession (Tsodyks et al., 1996; Jensen and Lisman, 1996a; Wallenstein and Hasselmo, 1997). In these models, entry to location 1 causes the read-out of locations 2-3-4-5. As shown in Figure 4, if one observes the response of a single cell (coding for location 5), it will initially occur late in theta at the end of the read-out sequence, and as the rat moves through the locations 2,3 , and 4 , it will move to earlier phases until it is driven by sensory input at the start of the cycle. The model presented later also performs read-out of sequences, but uses an interaction of forward associative retrieval and context-dependent gating of retrieval to address problems with the older model. In particular, this new model can keep the input present during the full theta cycle. In addition, previous models could not account for the fact that theta phase precession appears on later runs on a linear track, but does not appear as strongly on the first run of a day (Mehta et al., 2002), and place cell firing fields show a backward shift that occurs anew on each day of testing (Mehta et al., 1997). 

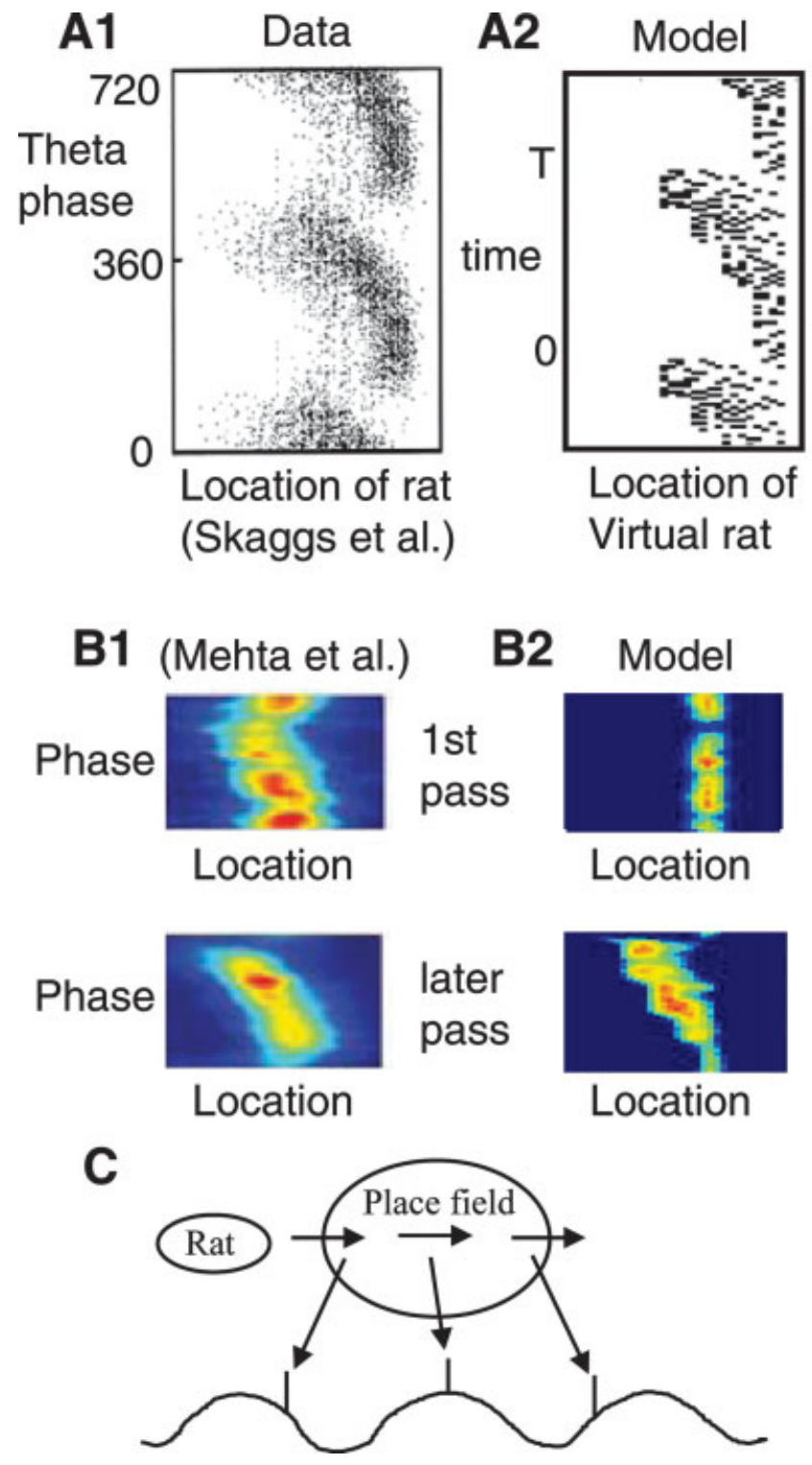

FIGURE 3. Theta phase precession of hippocampal place cells. A1: Experimental data (Skaggs et al., 1996) show that the theta phase of firing ( $y$-axis) moves to earlier phases as the rat moves from left to right ( $x$-axis). (Reprinted from Yamaguchi et al., 2002, J Neurophysiol 87:2629-2642, with permission from The American Physiological Society.) A2: The simulation shows the same pattern for activation of a single place cell in different locations as the virtual rat moves in one direction around a rectangular track. Firing during entry to a place field occurs when it is retrieved as a later portion of the retrieved sequence at late phases of theta. Firing during exit from the place field occurs when afferent input drives spiking activity at early phases of theta. B1: Experimental data (Mehta et al., 2002) showing that theta phase precession is not strong on the first pass through a location on a given day (top), but is stronger on later passes through the same location (bottom). B2: Simulation showing that absence of temporal context on first pass results in absence of phase precession on the first pass (top). On later passes (bottom), temporal context results in increased theta phase precession. C: Schematic illustration of phase precession. As the rat enters the place field of a cell, the cell fires at late phases of theta. Firing moves to earlier phases as the rat moves through the place field.

\section{Splitter cell responses}

Hippocampal neurons also show striking context-dependent changes in their response properties on a trial by trial basis during performance of a continuous spatial alternation task (Wood et al., 2000), as shown in Figure 5. These neurons have been called "splitter cells," though they could also be referred to as "episodic cells." During performance of a continuous spatial alternation task, these neurons fire in a trial selective manner as the rat runs up the stem of the maze. Even though the rat is running in the same direction in the same spatial location in the task, these splitter cells fire selectively depending on the prior or future response of the rat. For example, one particular splitter cell may fire only after a right turn response, but not after a left turn response. Further, analysis of these types of neurons in a different task (Ferbinteanu and Shapiro, 2003) demonstrates that the selectivity appears to depend more strongly on the previous location (retrospective response), though some cells show dependence on future location (prospective response).

\section{Buffering in EC}

The models presented here also address physiological data on the EC. This includes data showing theta rhythm oscillations in the EC (Alonso and Garcia-Austt, 1987a,b). In addition, data shows cholinergic modulation of intrinsic properties within the EC (Klink and Alonso, 1997). In slice preparations of the EC, perfusion with cholinergic agonists activates intrinsic calcium-sensitive cation currents that cause each spike to be followed by an afterdepolarization (Klink and Alonso, 1997). The influx of calcium during each spike activates the cation current, resulting in the afterdepolarization that causes repetitive generation of sustained spiking in the absence of synaptic input. This mechanism was proposed to interact with theta rhythm to provide a short-term buffer for input activity in the LismanJensen model (Lisman and Idiart, 1995; Jensen and Lisman, 1996b), and has been used in the models described later (Fransén et al., 2002; Koene et al., 2003; Hasselmo and Eichenbaum, 2005).

\section{Interactions With Prefrontal Cortex}

Recent physiological data have demonstrated that the firing of action potentials of neurons in prefrontal cortex shows a clear phase relationship to the hippocampal theta rhythm (Hyman et al., 2002, 2005; Hyman and Hasselmo, 2004; Manns et al., 2000a,b; Siapas et al., 2005). The computational modeling of behavior provides a functional framework for understanding the necessity of these phase relationships between activity in the hippocampus and prefrontal cortex.

\section{MODELS LINKING BEHAVIOR TO PHYSIOLOGY}

Linking behavior to physiology requires explicit modeling of both the memory-guided actions of a rat during behavior, and the physiological mechanisms underlying these actions. Network simu- 
lations of cortical structures have been used to guide the movements of a virtual rat in a virtual environment (Hasselmo et al., 2002a,c; Cannon et al., 2003; Koene et al., 2003; Hasselmo, 2005; Hasselmo and Eichenbaum, 2005; Koene and Hasselmo, 2005), in tasks including spatial reversal (Hasselmo et al., 2002b) spatial alternation and linear tracks (Hasselmo and Eichenbaum, 2005), and delayed nonmatch to position (Hasselmo and Zilli, 2005). The behavior in these modeled tasks has been guided by network simulations, which effectively simulate features of the physiological data described in the previous section.

As shown in Figure 6, the structure of these simulations is based on the anatomy and physiology of the hippocampus, EC, and prefrontal cortex. As the virtual rat moves through the task, information about its state in the environment (Place) and its receipt of food reward (Reward) is sent from the virtual rat to the neural simulation. Note that this simplified virtual rat does not view any state other than its immediate location,

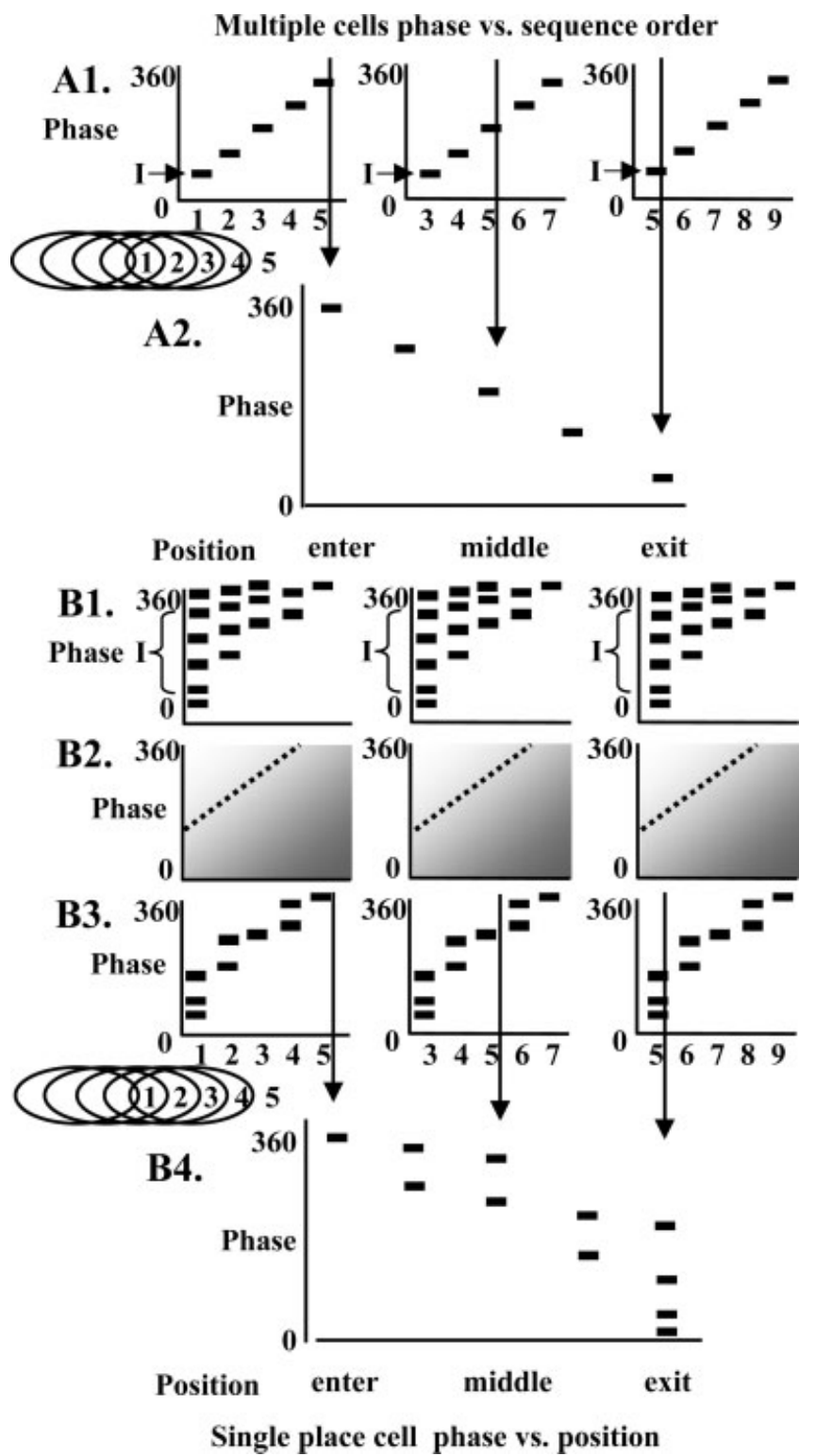

and so it only detects reward when it is received (in most tasks food reward is not visible at a distance). In the model, the prefrontal cortex performs goal-directed selection of next action (motor output) (Hasselmo, 2005; Koene and Hasselmo, 2005), performing functions similar to reinforcement learning algorithms (Sutton and Barto, 1998). Action selection depends on both the current state and episodic retrieval of previous responses from circuits representing hippocampus and EC (Hasselmo and Eichenbaum, 2005).

These models extend previous work that used network simulations of the hippocampal formation to guide the movements of a virtual rat in spatial tasks (Sharp et al., 1996; Burgess et al., 1997; Redish and Touretzky, 1998). These earlier simulations did not include a representation of the oscillatory dynamics of hippocampal theta rhythm during the behavior, though one model used assigned phases of theta firing to enhance the detail of place cell representation (Burgess et al., 1997). The series of models described here have progressed through a number of stages. Earlier models used associations between place cells in the hippocampus and EC to store pathways and select between possible pathways (Hasselmo et al., 2002c), similar to the hypothesis that Hebbian modification between place cells could provide a distance metric (Muller and Stead, 1996). However, later versions have used prefrontal cortical circuits to perform the action selection process, while the hippocampus itself performs encoding and retrieval of episodes. This focus on retrieval of episodes differs from models focused on the encoding and retrieval of single fixed patterns representing items such as words (Marr, 1971; Treves and Rolls, 1994; Hasselmo and Wyble, 1997; Norman and O'Reilly, 2003), and builds from previous models focused on encoding and retrieval

FIGURE 4. A. Simple model of phase precession based on retrieval of sequences (Tsodyks et al., 1996; Jensen and Lisman, 1996a). A1: Plot of multiple cells shows the retrieval of sequences of place cell activity over time at each new place in the environment. At place 1, places 2, 3, 4, and 5 are retrieved. At place 3, places $4,5,6$, and 7 are retrieved. Note that the model functions only if the input $I$ is present only at a single early phase. A2: When observing a single cell (5), firing initially appears late in the cycle, and then moves to earlier phases of theta. B: Theory of phase precession based on context-dependent retrieval of sequences (Hasselmo and Eichenbaum, 2005). B1: Forward retrieval in EC that spreads to more neurons as theta phase increases. Note that input is present at all phases of theta, but the length of forward spread increases during later phases. B2. Temporal context input from CA3 gates CA1 activity. Temporal context is stronger for more recent locations (to right), and weaker for more distant places (to left). For locations to the left of the dotted line, temporal context is too weak and CA1 activity falls below threshold. This input also decreases over phases within each cycle, causing activity to fall below threshold for more locations. B3: CA1 activity depends upon the multiplicative gating of EC input by temporal context from CA3. The input cue is present in EC and DG during the full cycle, but only results in retrieval activity in region CA1 when EC input converges with strong temporal context. B4: When observing a single cell (5), CA1 activity appears as wide, slightly scalloped distribution of spiking activity over phase and position. 
A1
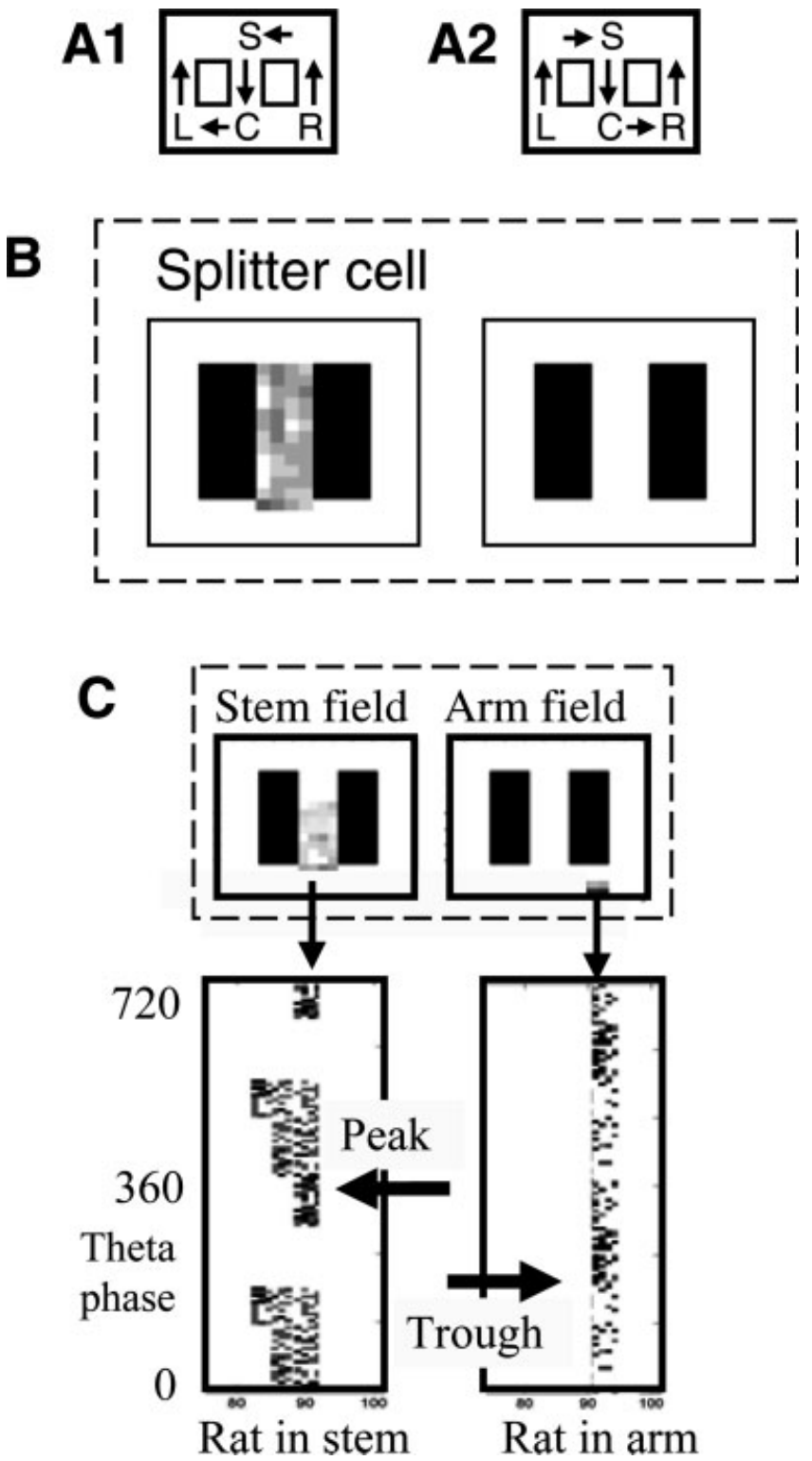

FIGURE 5. Simulation of "splitter cell" response. A: Behavioral context showing two trial types: A1: Post R-return from the right arm going to left, and A2: Post $\mathrm{L}-$ return from left arm going to right. B. A splitter cell representing the location just to the right of the choice point will fire as part of sequences retrieved in the stem after the virtual rat performs a right turn response (left side), but will not fire after a left turn response (right side). Gray scale represents number of spikes fired by simulations when virtual rat is in particular locations. Here, encoding is assumed to be induced only with dendritic spiking. C: Phase of neuronal firing for different places during spatial alternation. The splitter response in the stem occurs at late phases of theta, whereas the spiking activity during encoding in the response arm occurs at early phases of theta.

of full sequences (McNaughton and Morris, 1987; Levy, 1996; Jensen and Lisman, 1996a; Wallenstein and Hasselmo, 1997; Lisman, 1999; Hasselmo and Eichenbaum, 2005).

The latest model performs action selection in prefrontal cortex based on encoding and retrieval of episodic sequences, using the primary subregions of the hippocampal formation, including EC layer II, EC layer III, the dentate gyrus, and regions CA3 and CA1 of the hippocampus (Hasselmo and Eichenbaum, 2005). This network encodes episodes consisting of sequences of visits to different states (places) in the environment. Thus, encoding is based on sequential activation of "place cells" in the hippocampus (O'Keefe and Dostrovsky, 1971; O'Keefe, 1976; McNaughton et al., 1983; Muller et al., 1987; Eichenbaum et al., 1989; Muller and Kubie, 1989; Wiener et al., 1989; Skaggs et al., 1996; Wood et al., 2000; Huxter et al., 2003) and EC (Barnes et al., 1990; Frank et al., 2000). The construction of these models demonstrates the potential functional requirements for theta rhythm to time activity in multiple different regions during the encoding and retrieval required to perform memory-guided behavior. The following sections will briefly review four complementary and interacting hypotheses: (1) theta rhythm provides separation between encoding and retrieval dynamics (Hasselmo et al., 2002b), (2) theta rhythm enhances the context-dependent retrieval of previously encoded sequences (Hasselmo and Eichenbaum, 2005), (3) theta rhythm enhances the buffering of new input for encoding (Lisman and Idiart, 1995; Hasselmo et al., 2002a; Koene et al., 2003), and (4) theta rhythm times the interaction of prefrontal cortex action selection (Hasselmo, 2005; Koene and Hasselmo, 2005) with hippocampal retrieval (Hasselmo and Eichenbaum, 2005).

\section{Separation of Encoding and Retrieval}

Episodic memory function can run into problems if encoding is not separated from retrieval. For example, when you park your car in a large parking lot, you might recall parking your

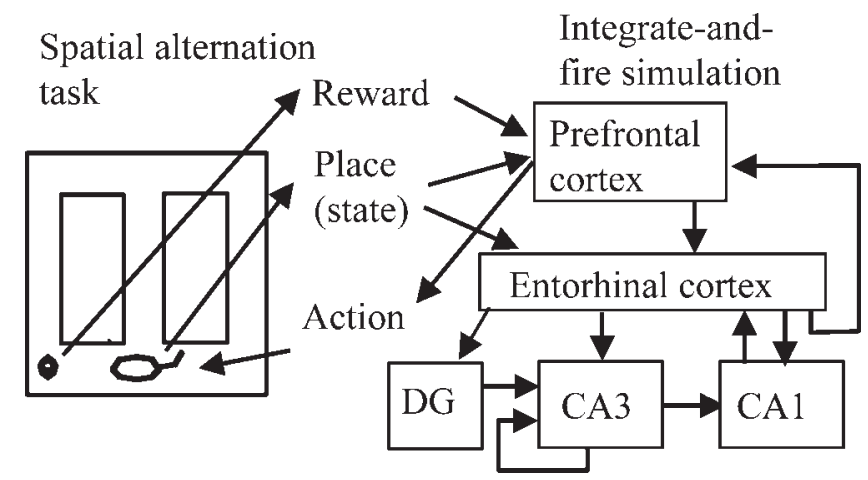

FIGURE 6. Overview of the neural simulation guiding actions of a virtual rat in a virtual spatial alternation task. The simulation receives input about the state of the virtual rat and its proximity to food reward, and guides the actions of the virtual rat based on prefrontal cortex activity. For spatial alternation, the prefrontal cortex can guide action selection based on selective sequential retrieval in the hippocampal formation of the previous response episode in the task. This sequential retrieval involves an interaction of forward associations in EC layer III (ECIII) that drive activity in region CA1. The retrieval activity in region CA1 is gated by the temporal context provided by circuits of the dentate gyrus (DG) and region CA3. This selective retrieval allows correct memoryguided selection of actions by the prefrontal cortex. 
car in a different location on a previous day. If you encode your retrieval of the previous episode as if it were new, then when you return to the parking lot you may go to the old parking location. This problem of confounding overlapping memories can be prevented by requiring a change in network dynamics during encoding and retrieval, as shown in many previous models (Hasselmo et al., 1992; Hasselmo, 1995). Selective presynaptic inhibition of glutamatergic transmission by muscarinic cholinergic receptors at association fiber synapses could provide this mechanism (Hasselmo and Schnell, 1994). The cholinergic suppression of transmission could suppress interference from retrieval while simultaneously enhancing encoding by increasing the induction of LTP. However, cholinergic modulation has a time course that appears to be too slow (Hasselmo and Fehlau, 2001). Alternately, the change in relative strength of encoding vs. retrieval could be provided by changes in synaptic currents during each cycle of the theta rhythm (Hasselmo et al., 2002b). This provides one mechanism for separation of these processes. Many other network models of associative memory function have used separate dynamics for encoding and retrieval, usually assuming clamping of activity during encoding (Anderson, 1972; Grossberg, 1975; McNaughton and Morris, 1987; Treves and Rolls, 1992).

Figure 2 summarizes how each cycle of the theta rhythm could contain separate phases of encoding and retrieval. These phases are defined relative to theta rhythm recorded in stratum lacunosum-moleculare of region CA1 (often referred to as "fissure theta"). The encoding phase occurs during strong synaptic input from EC, and is therefore at the trough of fissure theta. At this time, synaptic modification (LTP) is strong at synapses between neurons in region CA3 and between CA3 and CA1, but synaptic transmission at these same synapses is weak. This prevents retrieval of previously encoded associations from causing spurious associations between a new association and elements of an old association (Hasselmo et al., 2002b). In contrast, retrieval occurs during the peak and falling phase of hippocampal theta, when afferent input from EC is relatively weak. Retrieval requires strong synaptic transmission at recurrent collaterals in region CA3 and the Schaffer collaterals from CA3 to CA1, but to prevent this retrieval activity from causing interference, synaptic modification at these same synapses must be weak during this phase. The elements of this hypothesis are consistent with available experimental data, as summarized in the section on physiological data.

This framework can account for behavioral data showing that fornix lesions (which reduce theta rhythm) cause an increase in the number of errors after reversal in a T-maze task (M'Harzi et al., 1987). Specifically, rats with fornix lesions persist in visiting an arm that was previously rewarded but is currently unrewarded. This impairment could result from loss of theta rhythm, allowing the induction of LTP and synaptic transmission in stratum radiatum to be strong at the same time. After reversal, the rat makes erroneous visits to the previously rewarded location. In this case, strong synaptic transmission allows the rat to retrieve postsynaptic activity correspond- ing to memory of food at the now unrewarded location. This retrieval activity could cause further LTP, and thus strengthen associations with the memory of food despite the fact that the location is now unrewarded. This mechanism could slow the extinction of the old association and increase the period of error generation before reversal (Hasselmo et al., 2002b). The behavioral deficits following fornix lesions might also result from loss of slow modulatory effects of acetylcholine, but appear to depend on combined block of both cholinergic and GABAergic input (Pang et al., 2001).

\section{Context-Dependent Retrieval of Sequences}

Episodic memory requires selective retrieval of one memory without interference from other memories. For example, if you park in the same parking garage every day, it becomes difficult at the end of each day to remember where you parked your car. You must remember where you parked it this morning, without interference from multiple other memories of parking the car in different locations. The physiological changes during theta rhythm may enhance the selective context-dependent retrieval of individual encoded sequences without interference from other sequences (Sohal and Hasselmo, 1998a,b; Hasselmo and Eichenbaum, 2005). This hypothesis builds from earlier models of sequence encoding in hippocampal circuits. Marr (1971) initially proposed that excitatory recurrent connections in region CA3 could provide sequential associations between individual patterns in a sequence, and this mechanism has been used in many models (McNaughton and Morris, 1987; Blum and Abbott, 1996; Levy, 1996; Jensen and Lisman, 1996a; Wallenstein and Hasselmo, 1997; Lisman, 1999; Hasselmo and Eichenbaum, 2005). During encoding in these models, each pattern in a sequence activates a set of neurons shortly before the next pattern, and spike timing dependent plasticity (STDP) (Levy and Steward, 1983) strengthens synapses between the sequential patterns. During retrieval, input of the first pattern will cause activity to spread across strengthened synapses to cause sequential spiking in other patterns, reading out the full sequence (Levy, 1996; Wallenstein and Hasselmo, 1997). This simple sequence retrieval does not have to occur in region CA3. It could occur in any network in which retrieval output can cue another retrieval step. Thus, the same mechanism could occur at recurrent synapses in EC layer II or III (Hasselmo and Eichenbaum, 2005; Hasselmo et al., 2002c), in a loop involving dentate gyrus, region CA3 and mossy cells in the hilus (Lisman, 1999), or in a loop involving the full hippocampal circuit from EC back to EC. In addition, evidence suggests that the hippocampus may encode episodic sequences, whereas the basal ganglia may encode highly familiar sequences (White and McDonald, 2002).

Simple sequence encoding models cannot encode and selectively retrieve highly overlapping sequences (Levy, 1996). For example, consider the sequences A-B-C-D and E-B-C-F. Simple forward associations between each pattern would result in the cue "A" retrieving "B" and "C" followed by both "D" and "F" as the final pattern. Selective retrieval requires some additional 
mechanism for context-dependent retrieval of one out of many highly overlapping sequences, through gating of retrieval output by additional synaptic activity. In one network model of CA3, disambiguation of overlapping sequences has been obtained by having retrieval of the end of a sequence depend on synaptic input from persistent firing of additional CA3 neurons termed local context units (Levy, 1996). As an alternative, contextdependent retrieval could also be obtained by gating the output of retrieval, with input from another region. For example, the retrieval of region CA3 could be gated by activity from ECII, or output from CA3 to CA1 could be gated by activity from ECIII. In the model presented here, output from ECIII to CA1 is gated by context activity in the dentate gyrus and region $\mathrm{CA} 3$.

These mechanisms of context-dependent retrieval require a balance between the forward sequence retrieval and gating by context. Theta rhythm could provide a mechanism for sampling across different magnitudes of network variables. Once sequence retrieval activity occurs, feedback inhibition can ensure that the first, best matching sequence is selectively retrieved. Early models of this process used phasic changes in magnitude of synaptic transmission to allow retrieval of single associations because of a global context signal representing specific environmental cues selective for one episode (Sohal and Hasselmo, 1998a,b). The simulations of episodic retrieval reviewed here provide a more detailed model of the role of theta rhythm in allowing global context to regulate selective retrieval (Hasselmo and Eichenbaum, 2005).

Figure 7 (and Fig. 4B) summarize the mechanism in this most recent model (Hasselmo and Eichenbaum, 2005). As an example of overlapping behavioral sequences, consider the spatial alternation task. On each trial, the rat must retrieve memory of its previous response, so as to generate a response to the opposite arm of the maze. The hippocampal circuit mediates encoding and retrieval of the episodic memory of the previous trial (e.g., previous trial was left), which involves a sequence of states, going from the base (B) to the stem (S) to choice point (C) to the left arm (L). Correct behavior requires that the recent sequence B-S-C-L must be retrieved separately from the earlier sequence B-S-C-R involving the same locations but ending in the right arm of the maze. When the rat is at location $\mathrm{B}$, forward associations in EC will retrieve both the sequences ending in $\mathrm{L}$ and $\mathrm{R}$. The context-dependent retrieval of a single episode is obtained by gating the retrieval of forward associations with activity representing temporal context.

The model uses global temporal context (Howard and Kahana, 2002; Howard et al., 2004) to disambiguate the most recent sample trial (e.g., B-S-C-L) from other recent trials. The global temporal context consists of activity specific to individual segments of time. The model forms distinct representations for discrete times in the sequence of behavior and associates this temporal context with prior elements experienced during behavior. For example, the temporal context for the current visit to the base (CB) will form associations with prior states, and these associations are stronger for more recently experienced states (e.g., CB-L > CB-C > CB-S > CB-B > CB-R).
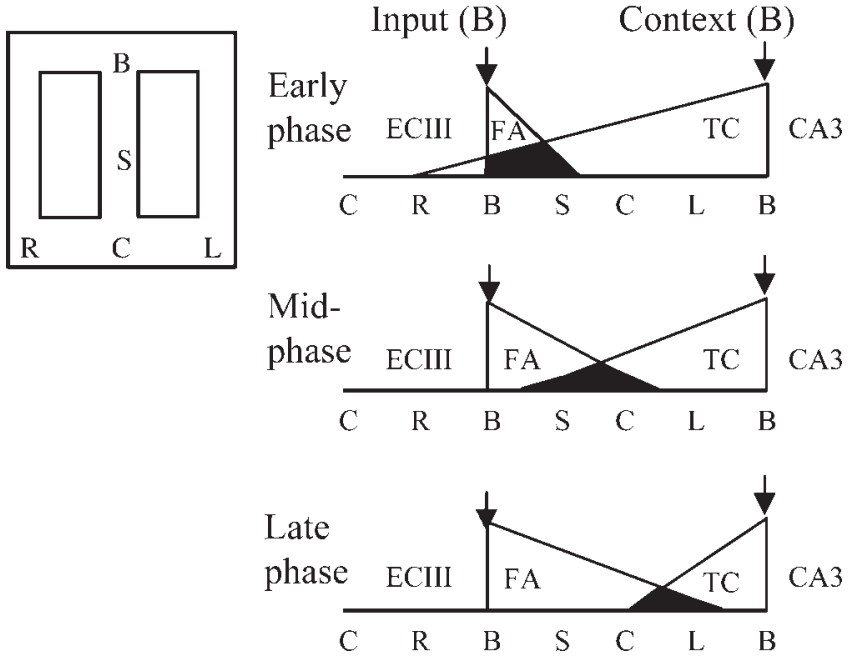

FIGURE 7. Example of context-dependent retrieval. Left: During spatial alternation, the virtual rat moves through the maze locations designated as base $B$, stem $S$, choice point $C$, and left arm $L$ or right arm $R$. Performance depends on retrieving memory of the prior response. The same input ( $B$, in this example) provides both the cue for forward associations (FA) and the context for temporal context (TC). In this example, the rat is at location B and retrieves B-S-C-L (retrieval of the previously experienced B-S$\mathrm{C}-\mathrm{R}$ would also occur, but is not shown). The width of triangles indicates the amount of spread of activity through populations of neurons in each region. Triangles in center indicate retrieval of forward associations (FA) in EC layer III (ECIII). Retrieval of temporal context (TC) in CA3 is represented by triangles on right. Black triangles indicate CA1 activity driven by multiplicative interactions of ECIII and CA3 input to CA1 (Spruston, pers. comm.). Top: At an early phase of theta, forward associations are weak and temporal context is strong (because of differences in depolarization in CA3 and ECIII). Their interaction (black) allows retrieval of the start of the sequence. Middle: Later, the spread across forward associations becomes stronger allowing retrieval of the middle of the sequence. Bottom: Near the end of the cycle, FA are very strong, and context is weaker, allowing retrieval of the end of the sequence with magnitude similar to activity at the start of the sequence.

Thus, associations with a particular context (e.g., base B) are weaker for states experienced at a longer time interval before the context, as shown schematically in Figures $4 \mathrm{~B} 2$ and 7. Region CA1 receives convergent input from EC and from temporal context mediated by dentate gyrus and region CA3. This allows selective retrieval of the recent sequence, because the forward retrieval of the recent sequence times context, C-L*(CB$\mathrm{L})$, has greater activity than the other sequence times context, $\mathrm{C}-\mathrm{R}^{*}(\mathrm{CB}-\mathrm{R})$. In the model, a phasic increase in forward retrieval is coupled with a gradual decrease in the backward, context-dependent retrieval, ensuring that each element of the sequence has the same retrieval strength (but the more recent sequence is consistently stronger than the more distant sequence). These changes can be obtained through phasic changes in magnitude of depolarization and synaptic transmission, which selectively allow the forward spread of activity to increase at the same time that the backward, context-dependent spread of activity weakens. 
Temporal context in the model uses dentate gyrus and region CA3. The dentate gyrus sets up distinct temporal representations, based on the highly divergent connections from EC to dentate gyrus (McNaughton, 1991; O’Reilly and McClelland, 1994). These distinct dentate representations can drive spiking of CA3 neurons via the mossy fibers. The active CA3 neurons can then form graded synaptic connections with neurons receiving input via perforant pathway projections from EC layer II. These connections can have graded strength because of exponential falloff of STDP with spikes generated at longer intervals by the buffer, or by different firing rates for more distant times. These graded connections can thereby encode an association between the distinct time representation and a graded representation of previous input activity. STDP could also provide graded strength of connections from EC to dentate gyrus (Levy and Steward, 1983), allowing prior states to selectively retrieve different context representations.

This process must deal with the problem that memories must be retrieved over a range of different temporal delays. The hippocampal theta rhythm provides a solution to this problem by allowing scanning for the first good match by phasically increasing context input because of changes in postsynaptic depolarization (Fox, 1989), or strength of excitatory synaptic transmission (Wyble et al., 2000). The activity would then be read-out by a multiplicative interaction of increasing entorhinal input and decreasing CA3 input, resulting in sequential retrieval that is equivalent in magnitude for each element of a sequence, but different in magnitude for different sequences, and strongest for the sequence best matching the current context (Hasselmo and Eichenbaum, 2005).

\section{Theta phase precession}

This model of the context-dependent retrieval process can replicate a number of physiological properties of single unit responses. The retrieval of sequences at each location of the virtual rat replicates the phenomenon of theta phase precession (O'Keefe and Recce, 1993; Skaggs et al., 1996). Previously, retrieval of sequences has been used to model the phenomenon of theta phase precession (Tsodyks et al., 1996; Jensen and Lisman, 1996a; Wallenstein and Hasselmo, 1997). In these models, entry to location 1 causes the read-out of locations 2-3-4-5. As shown in Figure 4, if one observes the response of a single cell (coding for location 5), it will initially occur late in theta at the end of the read-out sequence, and as the rat moves through the locations 2,3 , and 4 , it will move to earlier phases until it is driven by sensory input at the start of the cycle. Models shown in Figure $4 \mathrm{~A}$ require that input for the start of the sequence occurs only at one phase of the theta cycle. In contrast, the model of the context-dependent retrieval process can keep the input cue present during the full theta cycle (Hasselmo and Eichenbaum, 2005), as shown in Figures 3 and $4 \mathrm{~B}$. The phase of firing of region CA1 neurons in the new model depends on the relative strength of forward association retrieval (Fig. 4B1) and the backward temporal context retrieval (Fig. 4B2) at different phases of theta, as shown in Figures $4 \mathrm{~B}$ and 7 . In addition, the forward retrieval in EC occurs only during later phases of the theta cycle. During earlier phases, EC responds primarily to afferent input, resulting in a firing response for current location across a broad range of phases (appearing as an increase in phase variance in later portions of the place field).

As shown in Figure 3B, the context-dependent retrieval mechanism automatically addresses a previously puzzling property of theta phase precession, which other models do not address. In experimental data, theta phase precession is initially weak and becomes more prominent over initial trials on each day (Mehta et al., 2002). In our simulations, temporal context is weak on the first run because of the lack of previous exposure to the task on that day. The absence of the temporal context input from region CA3 to region CA1 neurons leaves only the input from forward associations in EC. This EC input is strongest for the current input, and therefore activity in region CA1 primarily reflects current input, resulting in no sequence retrieval and therefore no precession. As the rat moves through the task, it encounters further locations that provide strong temporal context for later trials, resulting in stronger temporal context input to region CA1 neurons, which allows sequential retrieval in CA1 that causes theta phase precession (Hasselmo and Eichenbaum, 2005). This same context mechanism could underlie the backward expansion of place fields observed on each day of recording (Mehta et al., 1997).

As shown in Figure 5, the model also simulates the phenomenon of some "splitter cells," shown in experimental data, which fire on the stem only for a specific trial ( $\mathrm{L}$ vs. R) even though all the cues are the same (Wood et al., 2000). This effect appears in the virtual rat because of selective retrieval on the stem of only the most recently performed sequence. In the model, this occurs for neurons that encode a location in one arm (e.g., the right arm). When sequence retrieval of the most recent episode occurs on the stem, the sequence will activate these neurons selectively, for example, after a right turn response, but not after a left turn response (Hasselmo and Eichenbaum, 2005).

The computational models presented here have generated a number of new predictions about the timing of spikes relative to hippocampal theta rhythm in behavioral tasks. Experiments currently underway in the Hasselmo and Eichenbaum laboratories are testing the prediction that splitter cell responses occur during the retrieval phase of theta rhythm oscillations (Griffin et al., 2005), as shown in Figure 5C. Thus, they should occur during the late phases of theta during which precession occurs. In contrast, splitter cells should reflect activity encoded in one arm of the maze on the preceding trial, thus, encoding activity should occur during the early encoding phase of theta rhythm. This same prediction applies to a delayed nonmatch to position task, in which firing on the sample trial (forced choice of one arm) should occur during the encoding phase, whereas firing on the test trial (both arms open for choice) should occur during the retrieval phase of theta rhythm. Simulations also demonstrate an interesting need to separate sequences of rewarded behavior into separate epochs. This separation could be provided by the suppression of theta rhythm during the receipt of 
reward, which has been described in both operant and spatial tasks (Wyble et al., 2004).

The mechanism of theta phase precession and splitter cells proposed here should also be relevant to the encoding and retrieval of sequences of odor stimuli. In a separate project, recording during an odor sequence disambiguation task (Agster et al., 2002) will test whether individual cells show theta phase precession of odor responses in which the phase of firing of a neuron will move to earlier phases of theta as the sequence progresses (activity will be plotted vs. odor number, rather than position in a place field). In addition, this same task can be used to test for splitter cell phenomena during the overlapping component of the odor sequence, analogous to splitter cell phenomena during the overlapping spatial component (the stem) of the spatial alternation task. In a delayed matching task responses to odors should occur on different phases during the sample period and test periods, or during match trials (which involve a previously encountered odor) vs. nonmatch trials. The generation of such predictions provides an opportunity to test and modify the models to address the full range of experimental data.

\section{Buffering of Input for Encoding}

Behavioral transitions in the environment occur over a time course of several hundred milliseconds or even seconds. For example, a rat may take several seconds to complete a full trial in a behavioral task. The time course of behavior is much slower than the time intervals important for STDP in the hippocampus, which results in strengthened synapses only when a presynaptic spike precedes a postsynaptic spike by less than about 40 ms. (Levy and Steward, 1983; Bi and Poo, 1998). A rat does not move very far in less than $40 \mathrm{~ms}$, and so the input from the environment does not change very much, raising the question of how a rat could form associations between neurons spiking at much slower intervals during sequential visits over longer periods in a task.

To hold information about prior location over hundreds of milliseconds or longer, encoding of sequences in the model uses buffering of input activity based on the Lisman-Jensen model (Lisman and Idiart, 1995; Jensen and Lisman, 1996b). Simulations have shown how buffering could result from input eliciting sustained spiking activity in the EC (Fransén et al., 2002; Hasselmo et al., 2002a; Koene et al., 2003), where cholinergic modulation activates afterdepolarization currents (Klink and Alonso, 1997) that allow rhythmic reactivation of the elements of the input sequence. Thus, theta rhythm in EC of these models serves to time the reactivation and updating of the working memory buffer. This buffer maintains place representations for a period of time sufficient for synapses to be modified between place cells activated by adjacent locations, forming the basis for episodic memory of specific sequences traversed through the environment.

\section{Action Selection in Prefrontal Cortex}

Lesions that impair hippocampal theta rhythm do not prevent general goal-directed behavior, but only specifically the memory-guided aspects of behavior. For example, in spatial reversal, fornix lesions do not impair learning of the initial reward location, but impairs learning of the new reward location. Thus, general processes of action selection must be outside of the hippocampus, but should interact strongly with hippocampal function. The prefrontal cortex component of the model provides a link to data on the association of theta rhythm with voluntary movement (Bland and Oddie, 2001). The model of prefrontal cortex requires separate phases for the encoding of new associations between states and actions and for the spread of activity from the goal through previous associations during retrieval (Hasselmo, 2005; Koene and Hasselmo, 2005). In the spatial alternation task, strengthening of synaptic connections in the prefrontal cortex forms associations between episodic memories retrieved by the hippocampus and the motor plans for specific actions. For example, a population of neurons in the prefrontal cortex responds to hippocampal activity for the memory of a previous left turn, this activity then spreads across strengthened synapses to activate prefrontal neurons representing a right turn response, which then activates the appropriate output. These interactions of hippocampus and prefrontal cortex require phasic timing of the input from hippocampus to prefrontal cortex. These timing requirements could be provided by the phasic timing of prefrontal cortex firing relative to the phase of theta rhythm in the hippocampus (Manns et al., 2000a,b; Hyman and Hasselmo, 2004; Hyman et al., 2002, 2005; Siapas et al., 2005).

\section{Acknowledgments}

I appreciate comments on the manuscript from Lisa Giocomo, Amy Griffin, Christina Rossi, Chantal Stern, and Eric Zilli.

\section{REFERENCES}

Aggleton JP, Neave N, Nagle S, Hunt PR. 1995. A comparison of the effects of anterior thalamic, mamillary body and fornix lesions on reinforced spatial alternation. Behav Brain Res 68:91-101.

Agster KL, Fortin NJ, Eichenbaum H. 2002. The hippocampus and disambiguation of overlapping sequences. J Neurosci 22:57605768.

Alonso A, Garcia-Austt E. 1987a. Neuronal sources of theta rhythm in the entorhinal cortex of the rat. I. Laminar distribution of theta field potentials. Exp Brain Res 67:493-501.

Alonso A, Garcia-Austt E. 1987b. Neuronal sources of theta rhythm in the entorhinal cortex of the rat. II. Phase relations between unit discharges and theta field potentials. Exp Brain Res 67:502-509.

Anderson JA. 1972. A simple neural network generating an interactive memory. Math Biosci 14:197-220.

Barnes CA, McNaughton BL, Mizumori SJ, Leonard BW, Lin LH. 1990. Comparison of spatial and temporal characteristics of neuronal activity in sequential stages of hippocampal processing. Prog Brain Res 83:287-300.

Berry SD, Seager MA. 2001. Hippocampal theta oscillations and classical conditioning. Neurobiol Learn Mem 76:298-313.

Berry SD, Thompson RF. 1978. Prediction of learning rate from the hippocampal electroencephalogram. Science 200:1298-1300. 
Bi GQ, Poo MM. 1998. Synaptic modifications in cultured hippocampal neurons: dependence on spike timing, synaptic strength, and postsynaptic cell type. J Neurosci 18:10464-10472.

Bland BH, Colom LV. 1993. Extrinsic and intrinsic properties underlying oscillation and synchrony in limbic cortex. Prog Neurobiol 41:157-208.

Bland BH, Oddie SD. 2001. Theta band oscillation and synchrony in the hippocampal formation and associated structures: the case for its role in sensorimotor integration. Behav Brain Res 127:119-136.

Blum KI, Abbott LF. 1996. A model of spatial map formation in the hippocampus of the rat. Neural Comput 8:85-93.

Bragin A, Jando G, Nadasdy Z, Hetke J, Wise K, Buzsaki G. 1995. Gamma $(40-100 \mathrm{~Hz})$ oscillation in the hippocampus of the behaving rat. J Neurosci 15:47-60.

Brankack J, Stewart M, Fox SE. 1993. Current source density analysis of the hippocampal theta rhythm: associated sustained potentials and candidate synaptic generators. Brain Res 615:310-327.

Burgess N, Donnett JG, Jeffery KJ, O'Keefe J. 1997. Robotic and neuronal simulation of the hippocampus and rat navigation. Philos Trans R Soc Lond B Biol Sci 352:1535-1543.

Buzsáki G. 2002. Theta oscillations in the hippocampus. Neuron 33:325-340.

Buzsáki G, Eidelberg E. 1983. Phase relations of hippocampal projection cells and interneurons to theta activity in the anesthetized rat. Brain Res 266:334-339.

Buzsáki G, Grastyan E, Czopf J, Kellenyi L, Prohaska O. 1981. Changes in neuronal transmission in the rat hippocampus during behavior. Brain Res 225:235-247.

Buzsáki G, Leung LW, Vanderwolf CH. 1983. Cellular bases of hippocampal EEG in the behaving rat. Brain Res 287:139-171.

Buzsáki G, Czopf J, Kondakor I, Kellenyi L. 1986. Laminar distribution of hippocampal rhythmic slow activity (RSA) in the behaving rat: current-source density analysis, effects of urethane and atropine. Brain Res 365:125-137.

Cannon RC, Hasselmo ME, Koene RA. 2003. From biophysics to behavior: Catacomb2 and the design of biologically-plausible models for spatial navigation. Neuroinformatics 1:3-42.

Csicsvari J, Hirase H, Czurko A, Mamiya A, Buzsaki G. 1999. Oscillatory coupling of hippocampal pyramidal cells and interneurons in the behaving Rat. J Neurosci 19:274-287.

Eichenbaum H, Wiener SI, Shapiro ML, Cohen NJ. 1989. The organization of spatial coding in the hippocampus: a study of neural ensemble activity. J Neurosci 9:2764-2775.

Ennaceur A, Neave N, Aggleton JP. 1996. Neurotoxic lesions of the perirhinal cortex do not mimic the behavioural effects of fornix transection in the rat. Behav Brain Res 80:9-25.

Ferbinteanu J, Shapiro ML. 2003. Prospective and retrospective memory coding in the hippocampus. Neuron 40:1227-1239.

Fox SE. 1989. Membrane potential and impedance changes in hippocampal pyramidal cells during theta rhythm. Exp Brain Res 77: 283-294.

Fox SE, Wolfson S, Ranck JB Jr. 1986. Hippocampal theta rhythm and the firing of neurons in walking and urethane anesthetized rats. Brain Res 62:495-508.

Frank LM, Brown EN, Wilson M. 2000. Trajectory encoding in the hippocampus and entorhinal cortex. Neuron 27:169-178.

Fransén E, Alonso AA, Hasselmo ME. 2002. Simulations of the role of the muscarinic-activated calcium-sensitive nonspecific cation current INCM in entorhinal neuronal activity during delayed matching tasks. J Neurosci 22:1081-1097.

Fujita Y, Sato T. 1964. Intracellular records from hippocampal pyramidal cells in rabbit during theta rhythm activity. J Neurophysiol 27: 1011-1025.

Givens B. 1996. Stimulus-evoked resetting of the dentate theta rhythm: relation to working memory. Neuroreport 8:159-163.

Givens B, Olton DS. 1994. Local modulation of basal forebrain: effects on working and reference memory. J Neurosci 14:3578-3587.
Givens BS, Olton DS. 1990. Cholinergic and GABAergic modulation of the medial septal area: effect on working memory. Behav Neurosci 104:849-855.

Golding N, Staff N, Spruston N. 2002. Dendritic spikes as a mechanism for cooperative long-term potentiation. Nature 418:326-331.

Green JD, Arduini AA. 1954. Hippocampal electrical activity and arousal. J Neurophysiol 17:533-557.

Griffin AL, Asaka Y, Darling RD, Berry SD. 2004. Theta-contingent trial presentation accelerates learning rate and enhances hippocampal plasticity during trace eyeblink conditioning. Behav Neurosci 118:403-411.

Griffin AL, Lee I, Eichenbaum H, Hasselmo ME. 2005. Phase relationship between single unit firing in CA1 and theta rhythm on a continuous T-maze alternation task. Abstr Soc Neurosci 31:72.7.

Grossberg S. 1975. A neural model of attention, reinforcement, and discrimination learning. Int Rev Neurobiol 18:263-327.

Hasselmo ME. 1995. Neuromodulation and cortical function: modeling the physiological basis of behavior. Behav Brain Res 67:1-27.

Hasselmo ME. 2005. A model of prefrontal cortical mechanisms for goal directed behavior. J Cogn Neurosci 17:1115-1129.

Hasselmo ME, Eichenbaum H. 2005. Hippocampal mechanisms for the context-dependent retrieval of episodes. Neural Netw, in press.

Hasselmo ME, Fehlau BP. 2001. Differences in time course of ACh and GABA modulation of excitatory synaptic potentials in slices of rat hippocampus. J Neurophysiol 86:1792-1802.

Hasselmo ME, Schnell E. 1994. Laminar selectivity of the cholinergic suppression of synaptic transmission in rat hippocampal region CA1: computational modeling and brain slice physiology. J Neurosci 14:3898-3914.

Hasselmo ME, Wyble BP. 1997. Free recall and recognition in a network model of the hippocampus: simulating effects of scopolamine on human memory function. Behav Brain Res 89:1-34.

Hasselmo ME, Zilli E. 2005. Hebbian synaptic modification in cortical circuits and memory-guided behavior in spatial alternation and delayed non-match to position. In: International Joint Conference on Neural Networks, Montreal, Canada: IEEE Press.

Hasselmo ME, Anderson BP, Bower JM. 1992. Cholinergic modulation of cortical associative memory function. J Neurophysiol 67: $1230-1246$.

Hasselmo ME, Cannon RC, Koene RA. 2002a. A simulation of parahippocampal and hippocampal structures guiding spatial navigation of a virtual rat in a virtual environment: a functional framework for theta theory. In: Witter MP, Wouterlood FG, editors. The parahippocampal region: organisation and role in cognitive functions. Oxford: Oxford University Press. p 139-161.

Hasselmo ME, Bodelon C, Wyble BP. 2002b. A proposed function for hippocampal theta rhythm: separate phases of encoding and retrieval enhance reversal of prior learning. Neural Computation 14: 793-817.

Hasselmo ME, Hay J, Ilyn M, Gorchetchnikov A. 2002c. Neuromodulation, theta rhythm and rat spatial navigation. Neural Netw 15: 689-707.

Holscher C, Anwyl R, Rowan MJ. 1997. Stimulation on the positive phase of hippocampal theta rhythm induces long-term potentiation that can be depotentiated by stimulation on the negative phase in area CA1 in vivo. J Neurosci 17:6470-6477.

Howard MW, Kahana MJ. 2002. A distributed representation of temporal context. J Math Psychol 46:269-299.

Howard MW, Fotedar MS, Datey AS, Hasselmo M. 2004. The temporal context model in spatial navigation and relational learning: explaining medial temporal lobe function across domains. Psychol Rev 112:75-116.

Huerta PT, Lisman JE. 1995. Bidirectional synaptic plasticity induced by a single burst during cholinergic theta oscillation in CA1 in vitro. Neuron 15:1053-1063.

Huxter J, Burgess N, O'Keefe J. 2003. Independent rate and temporal coding in hippocampal pyramidal cells. Nature 425:828-832. 
Hyman JM, Hasselmo M. 2004. Medial prefrontal cortex cells fire with a phase relationship to the hippocampal theta rhythm. Abstr Soc Neurosci 30:551.12.

Hyman JM, Wyble BP, Rossi CA, Hasselmo ME. 2002. Coherence between theta rhythm in rat medial prefrontal cortex and hippocampus. Abstr Soc Neurosci 28:477.6.

Hyman JM, Wyble BP, Goyal V, Rossi CA, Hasselmo M. 2003. Stimulation in hippocampal region CA1 in behaving rats yields LTP when delivered to the peak of theta and LTD when delivered to the trough. J Neurosci 23:11725-11731.

Hyman JM, Zilli EA, Paley AM, Hasselmo ME. 2005. Medial prefrontalcortex cells show dynamic modulation with the hippocampal theta rhythm dependent on behavior. Hippocampus 15:736-749.

Jensen O, Lisman JE. 1996a. Hippocampal CA3 region predicts memory sequences: accounting for the phase precession of place cells. Learn Mem 3:279-287.

Jensen O, Lisman JE. 1996b. Novel lists of $7+/-2$ known items can be reliably stored in an oscillatory short-term memory network: interaction with long-term memory. Learn Mem 3:257-263.

Kamondi A, Acsady L, Wang XJ, Buzsáki G. 1998. Theta oscillations in somata and dendrites of hippocampal pyramidal cells in vivo: activity-dependent phase-precession of action potentials. Hippocampus 8:244-261.

Klink R, Alonso A. 1997. Muscarinic modulation of the oscillatory and repetitive firing properties of entorhinal cortex layer II neurons. J Neurophysiol 77:1813-1828.

Koene RA, Gorchetchnikov A, Cannon RC, Hasselmo ME. 2003. Modeling goal-directed spatial navigation in the rat based on physiological data from the hippocampal formation. Neural Netw 16: 577-584.

Koene RA, Hasselmo ME. 2005. An integrate and fire model of prefrontal cortex neuronal activity during performance of goal directed decision-making. Cerebr Cortex, in press.

Kramis R, Vanderwolf CH, Bland BH. 1975. Two types of hippocampal rhythmical slow activity in both the rabbit and the rat: relations to behavior and effects of atropine, diethyl ether, urethane, and pentobarbital. Exp Neurol 49:58-85.

Kunec S, Hasselmo ME, Kopell N. 2005. Encoding and retrieval in the CA3 region of the hippocampus: a model of theta phase separation. J Neurophysiol 94:70-82.

Lerma J, Garcia-Austt E. 1985. Hippocampal theta rhythm during paradoxical sleep. Effects of afferent stimuli and phase relationships with phasic events. Electroencephalogr Clin Neurophysiol 60:46-54.

Leung L-WS. 1984. Model of gradual phase shift of theta rhythm in the rat. J Neurophysiol 52:1051-1065.

Levy WB. 1996. A sequence predicting CA3 is a flexible associator that learns and uses context to solve hippocampal-like tasks. Hippocampus 6:579-590.

Levy WB, Steward O. 1983. Temporal contiguity requirements for long-term associative potentiation/depression in the hippocampus. Neuroscience 8:791-797.

Lisman JE. 1999. Relating hippocampal circuitry to function: recall of memory sequences by reciprocal dentate-CA3 interactions. Neuron 22:233-242.

Lisman JE, Idiart MA. 1995. Storage of $7+/-2$ short-term memories in oscillatory subcycles. Science 267:1512-1515.

Macrides FH, Eichenbaum H, Forbes WB. 1982. Temporal relationship between sniffing and limbic theta rhythm during odor discrimination reversal learning. J Neurosci 2:1705.

Manns ID, Alonso A, Jones BE. 2000a. Discharge profiles of juxtacellularly labeled and immunohistochemically identified GABAergic basal forebrain neurons recorded in association with the electroencephalogram in anesthetized rats. J Neurosci 20:9252-9263.

Manns ID, Alonso A, Jones BE. 2000b. Discharge properties of juxtacellularly labeled and immunohistochemically identified cholinergic basal forebrain neurons recorded in association with the electroencephalogram in anesthetized rats. J Neurosci 20:1505-1518.
Markowska AL, Olton DS, Murray EA, Gaffan D. 1989. A comparative analysis of the role of fornix and cingulate cortex in memory: rats. Exp Brain Res 74:187-201.

Marr D. 1971. Simple memory: a theory for archicortex. Philos Trans R Soc Lond B Biol Sci 262:23-81.

McCartney H, Johnson AD, Weil ZM, Givens B. 2004. Theta reset produces optimal conditions for long-term potentiation. Hippocampus 14:684-687.

McNaughton BL. 1991. Associative pattern completion in hippocampal circuits: new evidence and new questions. Brain Res Rev 16: $193-220$

McNaughton BL, Morris RGM. 1987. Hippocampal synaptic enhancement and information storage within a distributed memory system. Trends Neurosci 10:408-415.

McNaughton BL, Barnes CA, O'Keefe J. 1983. The contributions of position, direction, and velocity to single unit-activity in the hippocampus of freely-moving rats. Exp Brain Res 52:41-49.

Mehta MR, Barnes CA, McNaughton BL. 1997. Experience-dependent, asymmetric expansion of hippocampal place fields. Proc Natl Acad Sci USA 94:8918-8921.

Mehta MR, Lee AK, Wilson MA. 2002. Role of experience and oscillations in transforming a rate code into a temporal code. Nature 417:741-746.

M'Harzi M, Palacios A, Monmaur P, Willig F, Houcine O, Delacour J. 1987. Effects of selective lesions of fimbria-fornix on learning set in the rat. Physiol Behav 40:181-188.

Molyneaux BJ, Hasselmo ME. 2002. GABA(B) presynaptic inhibition has an in vivo time constant sufficiently rapid to allow modulation at theta frequency. J Neurophysiol 87:1196-1205.

Muller RU, Kubie JL. 1989. The firing of hippocampal place cells predicts the future position of freely moving rats. J Neurosci 9: 4101-4110.

Muller RU, Stead M. 1996. Hippocampal place cells connected by Hebbian synapses can solve spatial problems. Hippocampus 6:709-719.

Muller RU, Kubie JL, Ranck JB Jr. 1987. Spatial firing patterns of hippocampal complex-spike cells in a fixed environment. J Neurosci 7:1935-1950.

Norman KA, O'Reilly RC. 2003. Modeling hippocampal and neocortical contributions to recognition memory: a complementary-learning-systems approach. Psychol Rev 110:611-646.

Numan R, Quaranta JR Jr. 1990. Effects of medial septal lesions on operant delayed alternation in rats. Brain Res 531:232-241.

O'Keefe J. 1976. Place units in the hippocampus of the freely moving rat. Exp Neurol 51:78-109.

O’Keefe J, Dostrovsky J. 1971. The hippocampus as a spatial map. Preliminary evidence from unit activity in the freely-moving rat. Brain Res 34:171-175.

O'Keefe J, Nadel L. 1978. The hippocampus as a cognitive map. Oxford, UK: Oxford University Press.

O’Keefe J, Recce ML. 1993. Phase relationship between hippocampal place units and the EEG theta rhythm. Hippocampus 3:317330.

O’Reilly RC, McClelland JL. 1994. Hippocampal conjunctive encoding, storage, and recall: avoiding a trade-off. Hippocampus 4:661682.

Orr G, Rao G, Houston FP, McNaughton BL, Barnes CA. 2001. Hippocampal synaptic plasticity is modulated by theta rhythm in the fascia dentata of adult and aged freely behaving rats. Hippocampus 11:647-654.

Pang KC, Nocera R, Secor AJ, Yoder RM. 2001. GABAergic septohippocampal neurons are not necessary for spatial memory. Hippocampus 11:814-827.

Pavlides C, Greenstein YJ, Grudman M, Winson J. 1988. Long-term potentiation in the dentate gyrus is induced preferentially on the positive phase of theta-rhythm. Brain Res 439:383-387.

Rawlins JN, Feldon J, Gray JA. 1979. Septo-hippocampal connections and the hippocampal theta rhythm. Exp Brain Res 37:49-63. 
Redish AD, Touretzky DS. 1998. The role of the hippocampus in solving the Morris water maze. Neural Comput 10:73-111.

Rizzuto DS, Madsen JR, Bromfield EB, Schulze-Bonhage A, Seelig D, Aschenbrenner-Scheibe R, Kahana MJ. 2003. Reset of human neocortical oscillations during a working memory task. Proc Natl Acad Sci USA 100:7931-7936.

Rudell AP, Fox SE, Ranck JB Jr. 1980. Hippocampal excitability related to the phase of theta rhythm in urethanized rats. Brain Res 294: 350-353.

Rudell AP, Fox SE, Ranck JB Jr. 1984. Hippocampal excitability phase-locked to the theta rhythm in walking rats. Exp Neurol 68: 87-96.

Sainsbury RS, Harris JL, Rowland GL. 1987a. Sensitization and hippocampal type 2 theta in the rat. Physiol Behav 41:489-493.

Sainsbury RS, Heynen A, Montoya CP. 1987b. Behavioral correlates of hippocampal type 2 theta in the rat. Physiol Behav 39:513519.

Seager MA, Johnson LD, Chabot ES, Asaka Y, Berry SD. 2002. Oscillatory brain states and learning: impact of hippocampal theta-contingent training. Proc Natl Acad Sci USA 99:1616-1620.

Seidenbecher T, Laxmi TR, Stork O, Pape HC. 2003. Amygdalar and hippocampal theta rhythm synchronization during fear memory retrieval. Science 301:846-850.

Semba K, Komisaruk BR. 1984. Neural substrates of two different rhythmical vibrissal movements in the rat. Neuroscience 12:761774.

Sharp PE, Blair HT, Brown M. 1996. Neural network modeling of the hippocampal formation spatial signals and their possible role in navigation: a modular approach. Hippocampus 6:720-734.

Siapas AG, Lubenov EV, Wilson MA. 2005. Prefrontal phase locking to hippocampal theta oscillations. Neuron 46:141-151.

Skaggs WE, McNaughton BL, Wilson MA, Barnes CA. 1996. Theta phase precession in hippocampal neuronal populations and the compression of temporal sequences. Hippocampus 6:149-172.

Sohal VS, Hasselmo ME. 1998a. Changes in GABAB modulation during a theta cycle may be analogous to the fall of temperature during annealing. Neural Comput 10:869-882.

Sohal VS, Hasselmo ME. 1998b. GABA(B) modulation improves sequence disambiguation in computational models of hippocampal region CA3. Hippocampus 8:171-193.

Stewart M, Fox SE. 1990. Do septal neurons pace the hippocampal theta rhythm? Trends Neurosci 13:163-168.

Sutton RS, Barto AG. 1998. Reinforcement learning (adaptive computation and machine learning). Cambridge MA: MIT Press.
Treves A, Rolls ET. 1992. Computational constraints suggest the need for two distinct input systems to the hippocampal CA3 network. Hippocampus 2:189-199.

Treves A, Rolls ET. 1994. Computational analysis of the role of the hippocampus in memory. Hippocampus 4:374-391.

Tsodyks MV, Skaggs WE, Sejnowski TJ, McNaughton BL. 1996. Population dynamics and theta rhythm phase precession of hippocampal place cell firing: a spiking neuron model. Hippocampus 6:271-280.

Vanderwolf CH. 1969. Hippocampal electrical activity and voluntary movement in the rat. Electroencephalogr Clin Neurophysiol 26: 407-418.

Vanderwolf CH, Kramis R, Robinson TE. 1977. Hippocampal electrical activity during waking behaviour and sleep: analyses using centrally acting drugs. Ciba Found Symp 58:199-226.

Vertes RP, Kocsis B. 1997. Brainstem-diencephalo-septohippocampal systems controlling the theta rhythm of the hippocampus. Neuroscience 81:893-926.

Wallenstein GV, Hasselmo ME. 1997. GABAergic modulation of hippocampal population activity: sequence learning, place field development, and the phase precession effect. J Neurophysiol 78:393-408.

Whishaw IQ. 1972. Hippocampal electroencephalographic activity in the Mongolian gerbil during natural behaviours and wheel running and in the rat during wheel running and conditioned immobility. Can J Psychol 26:219-239.

Whishaw IQ, Vanderwolf CH. 1973. Hippocampal EEG, behavior: changes in amplitude and frequency of RSA (theta rhythm) associated with spontaneous and learned movement patterns in rats and cats. Behav Biol 8:461-484.

White NM, McDonald RJ. 2002. Multiple parallel memory systems in the brain of the rat. Neurobiol Learn Mem 77:125-184.

Wiener SI, Paul CA, Eichenbaum H. 1989. Spatial and behavioral-correlates of hippocampal neuronal-activity. J Neurosci 9:2737-2763.

Winson J. 1978. Loss of hippocampal theta rhythm results in spatial memory deficit in the rat. Science 201:160-163.

Wood ER, Dudchenko PA, Robitsek RJ, Eichenbaum H. 2000. Hippocampal neurons encode information about different types of memory episodes occurring in the same location. Neuron 27:623-633.

Wyble BP, Linster C, Hasselmo ME. 2000. Size of CA1-evoked synaptic potentials is related to theta rhythm phase in rat hippocampus. J Neurophysiol 83:2138-2144.

Wyble BP, Hyman JM, Rossi CA, Hasselmo M. 2004. Analysis of theta power in hippocampal EEG during bar pressing and running behavior in rats during distinct behavioral contexts. Hippocampus 14:368-384. 